PSI UNISC

Recebido em: 01/04/2018

Autor para contato: carine tbz@hotmail.com
ISSN: $2527-1288$

Aceito em: 02/07/2018

doi: $10.17058 /$ psiunisc.v2i2.11907

\title{
Intervenção Hospitalar Multiprofissional com Prematuros: uma Revisão Sistemática
}

Intervención Hospitalar Multidisciplinaria con Prematuros: una Revisión Sistemática

Multiprofessional Hospital Intervention with Premature Birth: a Systematic Review

Carine Tabaczinski

ORCID: https://orcid.org/0000-0002-9622-752X Faculdade Meridional, Rio Grande do Sul/Brasil

Denice Bortolin

ORCID: https://orcid.org/0000-0003-1891-1075 Faculdade Meridional, Rio Grande do Sul/Brasil

Luiz Ronaldo Freitas de Oliveira

ORCID: https://orcid.org/0000-0002-7600-8442 Faculdade Meridional, Rio Grande do Sul/Brasil

\section{Resumo}

O presente estudo objetivou mapear e compreender os estudos empíricos sobre a intervenção hospitalar multiprofissional com prematuros a fim de analisar como ocorrem essas intervenções e identificar a associação entre as ações desenvolvidas e o impacto gerado ao pré-termo, bem como, verificar a existência de intervenções hospitalares voltadas à interação mãe-bebê no contexto da prematuridade com enfoque na área da psicologia. Para isso, realizou-se uma busca e análise dos artigos, incluindo pesquisas nas bases eletrônicas Medline, Lilacs, Scielo, PubMed, Pepsic e BVS no mês de setembro de 2017. Foram incluídas publicações compreendendo o período de 2013 a 2017 com o uso de termos padronizados e previstos pelo DECS e Mesh Terms: premature birth, hospitals e health services. Além disso, foram incluídos artigos sobre intervenção hospitalar multiprofissional com prematuros, de qualquer natureza, com ações associadas à sua qualidade de vida e voltadas diretamente ao pré- termos. Dos 2040 artigos localizados, três foram selecionados de acordo com os critérios de inclusão adotados. A análise sistemática permitiu identificar a associação entre as ações desenvolvidas e o impacto destas intervenções benéficas, não farmacológicas, econômicas e não invasivas ao neonato e a possível relação destes achados com a construção corporal do ego do recémnascido. Discutem-se os achados dessa revisão com a literatura psicanalítica pertinente.

Palavras-chaves: Nascimento prematuro; Hospital; Áreas da saúde.

\section{Resumen}

El presente estudio objetivó mapear y comprender los estudios empíricos sobre intervención hospitalar multidisciplinaria con prematuros al cabo de analisar como ocurren esas intervenciones e identificar asociación entre las acciones desarolladas y el impacto generado al pretérmino, bien como, verificar la existencia de intervenciones hospitalares vueltas a la interacción madre-bebé en el contexto de la prematuridad con enfoque en la área de la psicología. Para eso, se realizó una búsqueda y análisis de los artículos incluyendo pesquisas en las bases electrónicas Medline, Lilacs, Scielo, PubMed, Pepsic y BVS en el mes de septiembre de 2017. Fueran incluídas publicaciones comprendiendo el período de 2013 hasta 2017 con el uso de términos padronizados y previstos por el DECS y Mesh Terms: premature birth, hospitals and health services. Artículos sobre intervención hospitalar multidisciplinaria con prematuros, de qualquier naturaleza, con acciones asociadas a su 
calidad de vida y vueltas directamente al pretérmino fueron incluídos. De los 2040 artículos localizados, tres fueron seleccionados de acuerdo con los criterios de inclusión adoptados. El análisis sistemático permitió identificar la asociación entre las acciones desarolladas y el impacto de estas intervenciones beneficas, no farmacologicas, económicas y no invasivas al neonato y a la posible relación de estes hallazgos con la construcción corporal del ego del recién nacido. Se discuten los hallazgos de esa revisión con la literatura psicanalítica pertinente.

Palabras claves: Nacimiento prematuro; Hospital; Áreas de la salud.

\begin{abstract}
The present study aimed to map understand the empirical studies on multiprofessional hospital intervention with premature birth, in order to identify how these interventions, occur and identify the association between the actions developed and the impact generated at the preterm period, as well as, to verify the existence of hospital interventions focused on mother-infant interaction in the context of prematurity with a focus on psychology. For this, a search and analysis of the articles including searches in the electronic databases Medline, Lilacs, Scielo, PubMed, PepSic and BVS were carried out in September 2017. Publications including the period from 2013 to 2017 were included, using standardized terms and provided by DECS and Mesh Terms: premature birth, hospitals and health services. Only articles about multiprofessional hospital intervention with preterm infants, of any kind, with actions attached to their quality of life and focus directly to the preterm were included. A total of 2040 articles were identified, of which only three were selected according to the inclusion criterion adopted. The systematic analysis allowed to identify the association among the actions developed and the impact of these beneficial, non-pharmacological, economic and non-invasive interventions to the neonate and the possible relationship of these findings with the body construction of newborn's ego. The findings of this review are discussed with the relevant psychoanalytic literature.
\end{abstract}

Keywords: Premature birth; Hospitals; Health services.

\section{Introdução}

As intervenções com bebês prematuros exigem da área da saúde atenção e pesquisas constantes. O Ministério da Saúde (2014) define como pré-termo toda criança nascida antes de completar 37 semanas ( $<259$ dias), em graus de prematuridade, classificados como: limítrofes (entre 35 e 36 semanas), os prematuros moderados (idade gestacional entre 31 e 34 semanas) e os prematuros extremos com idade gestacional menor ou igual a 30 semanas. Aproximadamente $1 \mathrm{em}$ cada 10 bebês nasce pré-termo e, mundialmente, a prematuridade é considerada a principal causa de óbitos em crianças menores de 5 anos (World Health Organization [WHO], 2017).

A média mundial de nascimentos prematuros é de $10 \%$, sendo que no Brasil essa taxa está acima, chegando a $12 \%$, o que faz do país o $10^{\circ}$ no ranking de nascimentos prematuros (Fundação Osvaldo Cruz [Fiocruz], 2016). Muitas vezes o nascimento prematuro está associado à hospitalização do bebê devido aos problemas de saúde que este apresenta (Shaffer, 2005). Neste sentido, este pode representar para os pais e para a criança um período complicado e angustiante, em que a relação e os cuidados iniciais, que poderiam ocorrer entre eles de maneira natural, são dificultados no ambiente da UTIN - Unidade de Tratamento Intensivo Neonatal (Duarte, Santos, Silva, Oliveira \& Sampaio, 2010).

Há uma alta incidência de partos prematuros, muitos deles seguidos de óbitos. Essa alta incidência de mortalidade em crianças pré-termo está relacionada, segundo os dados da Organização Mundial da Saúde (WHO, 2015), à falta de cuidados simples, 
efetivos e baratos, como proporcionar ao recém-nascido um ambiente de temperatura adequada (calor) e fornecer apoio à amamentação. A falta de cuidados básicos para combater infecções e problemas respiratórios também está relacionada às altas taxas de mortalidade em ambientes de baixa renda, diferente da realidade dos países desenvolvidos nos quais praticamente todos esses bebês sobrevivem (WHO, 2015).

A fim de minimizar os danos causados aos bebês decorrentes do nascimento prematuro, o Método Canguru surgiu como uma série de ações simples e econômicas para auxiliar no desenvolvimento e na qualidade de vida das crianças pré-termo. $\mathrm{O}$ Método consiste em vários fatores, que vão desde o acolhimento aos pais durante o pré-natal ou na UTIN, até a alta hospitalar seguido de acompanhamento ambulatorial e/ou em casa. A Posição Canguru integra o método e possibilita o contato pele a pele do bebê com o cuidador, estimulando, assim, a participação da mãe no cuidado ao neonato prematuro (Ministério da Saúde [MS], 2014).

O Programa iniciou na Colômbia, no Hospital San Juan de Dias - Instituto Materno Infantil (IMI), de Bogotá, em 1979. Seus idealizadores - Edgar Sanabria Rey e Héctor Martínez Gomes- buscavam uma intervenção para reduzir o elevado número de mortalidade entre os recém-nascidos prematuros (Carvalho \& Prochnik, 2001). Devido à escassez de recursos tecnológicos da época, os profissionais de saúde abrigavam dois ou três bebês juntos em uma mesma incubadora, o que agravava as possibilidades de infecções. A fim de diminuir o risco de infecções e de mortalidade, as mães passaram a ser mais atuantes e a substituir a tecnologia que faltava para dar conta da demanda. O método passou a suprir a falta desta mãe e, simultaneamente, reduzia a mortalidade neonatal e o tempo de permanência da díade mãe-bebê na maternidade (Charpak, Figueroa \& Hamel, 1999).

O Método Canguru tomou proporções internacionais e vem sendo adaptado em diferentes culturas e contextos. Este, foi projetado como um tratamento alternativo para bebês com baixo peso ao nascer e prematuros. Trata-se de uma intervenção de baixo custo que é acessível, simples e viável para a maioria das mães nas primeiras semanas pósparto (Cooijmans, Beijers, Rovers, \& Weerth, 2017). O contato pele a pele da Posição Canguru diminui os sintomas maternos de depressão pós-parto, melhora a saúde física e mental da mãe e o vínculo da díade (Souto, Jager, Pereira, \& Dias, 2014; Spehar \& Seidl, 2013; Rolim, Vidal, Mariano, Campos, \& Frota, 2008; Penalva \& Schwartzman, 2006). Melhora ainda a duração e a qualidade da amamentação (Moore, Bergman, Anderson, \& Medley, 2016; Sharma, 2016), o ganho de peso e o crescimento do recém-nascido prematuro, além de reduzir os custos hospitalares e o período de internação (Bilotti et al., 2016; Sharma, Murki, \& Oleti, 2016).

Dentre as intervenções no âmbito brasileiro destacam-se a aplicação do Manual Técnico da Atenção Humanizada ao RecémNascido de baixo peso (Método Canguru) (MS, 2014), citado anteriormente, a Rede Cegonha (MS, 2011) que assegura às mulheres e às crianças atenção humanizada, acesso a seus direitos no que diz respeito à gestação e suas nuances, e no crescimento e desenvolvimento saudável da criança. Recentemente, o Ministério da Saúde lançou o programa de Estratégia QualiNEO, com o objetivo de integrar outros programas já desenvolvidos pelo Ministério da Saúde voltados à saúde da criança (Portal da Saúde, 2017).

A literatura tem registrado uma gama de intervenções hospitalares multiprofissionais com prematuros, incluindo ações da fisioterapia que, utiliza da massoterapia como intervenção complementar para induzir o sono (Yates et al., 2014) e para manter estável o nível de bilirrubina em recém-nascidos prétermos (Basiri-Moghadam, Basiri-Moghadam, Kianmehr \& Jani, 2015). A psicologia também intervém com prematuros no contexto hospitalar. As intervenções estão voltadas ao impacto positivo na relação mãe- 
bebê, pois a criança que vivencia uma interação saudável com a mãe sente-se acolhida e torna-se um adulto seguro (Bowlby, 1990; Winnicott, 1988).

Além disso, há evidências de intervenções alternativas e não farmacológicas, como a musicoterapia, que é considerada uma opção de intervenção benéfica aos resultados fisiológicos e de alimentação, bem como para a redução do tempo de permanência na UTIN e do estresse dos pais (O'Toole, Francis \& Pugsley, 2017). Encontramos outro exemplo de intervenção alternativa e não farmacológica no estudo de Costa, Beleza, Souza e Ribeiro (2016), na qual o uso de rede em prematuros (pano posicionado dentro da incubadora, simulando o útero materno) proporcionou melhoras significativas no seu estresse se comparado ao uso do ninho (pano flexível em forma de "U" em torno do recém-nascido para contê-lo).

Partindo dessas constatações, o presente estudo teve como objetivo revisar sistematicamente os artigos científicos sobre o tema da intervenção hospitalar multiprofissional com prematuros a fim de analisar como ocorrem essas intervenções e identificar a associação entre as ações desenvolvidas e o impacto gerado ao prétermo, bem como, verificar a existência de intervenções hospitalares voltadas à interação mãe-bebê no contexto da prematuridade com enfoque na área da psicologia. As indagações sobre a temática surgem após uma experiência prática, durante o período de estágio clínico do último ano da faculdade de psicologia, realizado nos setores da Maternidade e UTIN de um Hospital Escola, vinculado à Instituição de Ensino Superior.

\section{Método}

Esta revisão sistemática da literatura foi desenvolvida de acordo com as diretrizes do Preferred Reporting Itens for Systematic Reviews and Meta- Analyses (Mother, Liberati, Tetzlaff, Altman, The PRISMA Group, 2009; $\quad$ http://www.prismastatement.org/.), que avalia as características de redação do estudo e não necessariamente sua qualidade metodológica, sendo composto por um conjunto 27 itens sobre informações que devem ser claramente descritas no artigo.

A identificação e a seleção dos artigos em todas as bases de dados foram realizadas simultaneamente por dois juízes independentes durante o mês de setembro 2017. Em caso de discordância entre os pares, um terceiro avaliador seria acionado para a arbitragem final, o que foi desnecessário. Os termos baseados nos descritores DECS e Mesh Terms foram: premature birth, hospitals e health services. Aqueles estudos que versavam sobre fundamentação teórica foram excluídos da análise.

\section{Critérios de inclusão e fontes de informação}

Dois critérios iniciais de inclusão foram adotados para a seleção: artigos completos de periódicos científicos, publicados nos últimos cinco anos, em inglês ou português, disponíveis de forma online e com acesso na íntegra gratuitamente. A revisão buscou estudos que avaliaram somente a intervenção direta com prematuros no contexto hospitalar, sendo que o conceito de prematuridade deveria atender aos critérios do Ministério da Saúde (MS).

\section{Estratégia de busca}

As bases de publicações eletrônicas foram a Medline, Lilacs, Scielo (campo Abstract), PubMed e Pepsic (campo Title/Abstract) e BVS, por estarem relacionadas à área da saúde. Utilizaram-se os seguintes descritores padronizados (apenas em inglês): premature birth, hospitals e health services. Utilizou-se a expressão booleana "AND" a fim de localizar os registros com ocorrência simultânea entre os descritores referidos.

\section{Seleção dos estudos}

A primeira etapa foi a de identificação dos estudos. Para fins de seleção, após a aplicação dos critérios de inclusão (ano de 
publicação, idioma e acesso), foram descartadas as publicações em duplicidade por meio da leitura dos títulos das mesmas. Para a elegibilidade, os textos foram lidos na íntegra. Foram excluídos relatos, produções oriundas de congressos (resumos, resumos expandidos ou textos completos), dissertações de mestrado, teses de doutorado, livros ou capítulos de livros, resenhas, comentários de artigos ou editoriais; os artigos com foco na intervenção hospitalar em recém-nascidos a termo; e destinados à intervenção hospitalar com prematuros nas áreas da saúde, porém com um enfoque que não é pertinente ao estudo.

\section{Processo de extração de dados}

Os estudos que contemplaram os critérios de inclusão foram analisados de forma descritiva e qualitativa, de acordo com as seguintes categorias: delineamento, objetivo e resultados. $\mathrm{Na}$ segunda etapa, foram analisadas as convergências e divergências entre as publicações, selecionando os temas mais recorrentes, os quais foram sistematizados em forma de tabela (tabela 2) e explanados ao longo da discussão.

\section{Risco de viés entre os estudos}

A identificação e a seleção dos artigos nas bases de dados foram feitas por dois pesquisadores de forma independente $\mathrm{e}$ sistemática, que fizeram a identificação inicial pelos títulos das publicações encontradas pelos descritores e, posteriormente, pelos resumos obtidos por busca eletrônica. Após essa seleção, uma nova avaliação foi feita pelos dois pesquisadores, que determinaram de forma consensual os estudos a serem lidos na íntegra e incluídos na revisão. Como os dois pesquisadores entraram em um consenso, não houve a necessidade da intervenção de um terceiro juiz.

\section{Resultados}

Foram localizados 2040 registros no mês de setembro de 2017 entre os dois pesquisadores. Após a aplicação dos filtros aos critérios de inclusão e identificação das duplicatas, 300 artigos foram selecionados e analisados por meio do título e do resumo. Das 19 publicações avaliadas para elegibilidade, selecionaram-se para análise apenas quatro artigos que se adequavam aos critérios estabelecidos para essa revisão sistemática, sendo um deles duplicata, como ilustra a Figura 1.

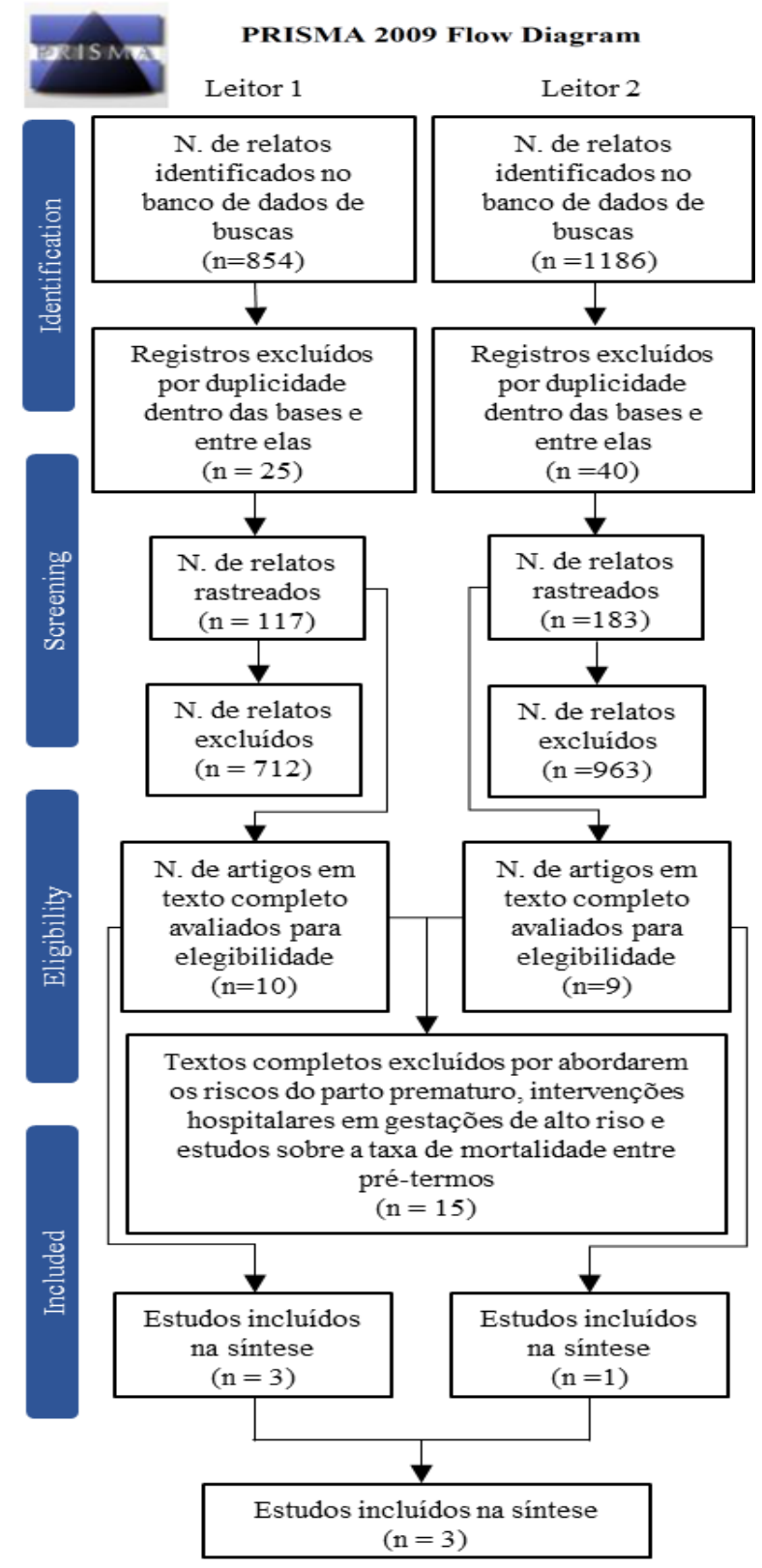

Figura 1. Fluxograma da seleção de artigos para a revisão sistemática

Fonte: Adaptado de "Principais itens para relatar Revisões sistemáticas e Meta-análises: A recomendação PRISMA" de T.F. Galvão., T de S. A. Pansani e D. Harrad, 2015, Epistemologia e Saúde, 24(2). doi: $10.5123 /$ S1679-49742015000200017 
Constatou-se que os artigos não diferem quanto ao tipo de delineamento, já que ambos são caracterizados como ensaios clínicos, de natureza experimental/qualitativo (Ahmed et al. [E2], 2015) design cruzado (Alinejad-Naeini, Mohagheghi, Peyrovi \& Mehran [E1], 2014) e randomizado (Saeidi, Ghorbani \& Moghadam [E3], 2015). Dos três artigos analisados, dois tiveram como objetivo intervenções voltadas ao ganho de peso do Tabela 1

Sintese dos artigos analisados pré-termo (E2; E3) e um avaliou o impacto da dor do neonato prematuro durante procedimentos de sucção, utilizando a Posição Facilitadora (E1). Todos os artigos analisados obtiveram resultados importantes no que se propuseram a avalizar, tratando-se de intervenções benéficas, não farmacológicas, econômicas e não invasivas ao neonato como mostra a Tabela 1 .

\begin{tabular}{|c|c|c|c|}
\hline Autor (ano) & Delineamento & Objetivo e Intervenção & Resultados \\
\hline $\begin{array}{l}\text { Alinejad- } \\
\text { Naeini, } \\
\text { Mohagheghi, } \\
\text { Peyrovi \& } \\
\text { Mehran } \\
(2014)(\mathrm{E} 1) \\
\end{array}$ & $\begin{array}{l}\text { Ensaio clínico } \\
\text { com desenho } \\
\text { cruzado. }\end{array}$ & $\begin{array}{l}\text { Avaliar o impacto da facilitated } \\
\text { tucking position (posicionamento } \\
\text { do recém-nascido, FTP) sobre a dor } \\
\text { comportamental durante a sucção } \\
\text { em neonatos prematuros. }\end{array}$ & $\begin{array}{l}\text { Observou-se a relação da facilitated tucking } \\
\text { position na diminuição da dor intensa dos } \\
\text { recém-nascidos pré- termos. Os resultados } \\
\text { evidenciam que a intervenção trata-se de um } \\
\text { método não- farmacológico e seguro nas } \\
\text { ações do manejo da dor durante a sucção. }\end{array}$ \\
\hline $\begin{array}{c}\text { Ahmed et al. } \\
(2015)(\mathrm{E} 2)\end{array}$ & $\begin{array}{l}\text { Ensaio clínico } \\
\text { experimental, } \\
\text { qualitativo. }\end{array}$ & $\begin{array}{l}\text { Determinar o efeito de } 7 \text { dias de } \\
\text { tactile kinesthetic simulation } \\
\text { (estimulação cinestésica tátil, TKS) } \\
\text { sobre o peso de bebês prematuros e } \\
\text { seu tempo de hospitalização. } \\
\end{array}$ & $\begin{array}{l}\text { Evidenciou-se o efeito benéfico da TKS sob } \\
\text { o ganho de peso dos recém-nascidos } \\
\text { prematuros, sua alta hospitalar antecipada e, } \\
\text { simultaneamente, a economia gerada. }\end{array}$ \\
\hline $\begin{array}{l}\text { Saeidi, } \\
\text { Ghorbani \& } \\
\text { Moghadam } \\
(2015)(E 3)\end{array}$ & $\begin{array}{l}\text { Ensaio clínico } \\
\text { randomizado. }\end{array}$ & $\begin{array}{l}\text { Investigar o efeito da massage with } \\
\text { Medium- Chain Triglyceride Oil } \\
\text { (massagem com óleo de } \\
\text { triglicerídeos de cadeia média, } \\
\text { MCT) no ganho de peso dos recém- } \\
\text { nascidos hospitalizados na UTIN. }\end{array}$ & $\begin{array}{l}\text { Os resultados apresentam uma eficácia } \\
\text { significativa massage with Medium- Chain } \\
\text { Triglyceride Oil no ganho de peso em recém- } \\
\text { nascidos prematuros em comparação ao } \\
\text { grupo de controle, sem causar. }\end{array}$ \\
\hline
\end{tabular}

Nota-se que os artigos incluídos se encontram na língua inglesa, consequentemente, a escassez de estudos na língua portuguesa, voltados ao prematuro é evidente. Ao se atentar para a cronologia, não foram encontrados estudos na temática nos últimos dois anos.
Outro dado importante diz respeito aos locais desses estudos. Duas das pesquisas foram realizadas em hospitais da capital (E1) e na segunda maior cidade do Irã (E3). Já o estudo de Ahmed et al. (2015) foi realizado na capital e segunda maior cidade da África, como ilustra a Tabela 2.

Tabela 2

Características dos estudos avaliados.

\begin{tabular}{|c|c|c|c|}
\hline País & População/amostra & $\begin{array}{c}\text { Tempo de } \\
\text { intervenção }\end{array}$ & Análise \\
\hline $\begin{array}{l}\text { Tehran, Iran } \\
\text { (Teerã, Irã) } \\
\quad(\mathrm{E} 1)\end{array}$ & $\begin{array}{l}34 \text { recém-nascidos } \\
\text { prematuros, peso } \\
\text { (1200g ou mais), } \\
\text { idade gestacional } \\
\text { entre } 29-37 \text { semanas. }\end{array}$ & $\begin{array}{c}5 \text { meses } \\
\text { (Jan./Maio. 2013). }\end{array}$ & $\begin{array}{l}\text { Coleta de dados: Formulário de características } \\
\text { demográficas e Perfil de Pretérmino da Dor } \\
\text { Infantil (PIPP). Análise: Pacote Estatístico para } \\
\text { Ciências Sociais (SPSS). T- test para o } \\
\text { pareamento das informações. }\end{array}$ \\
\hline $\begin{array}{l}\text { Khartoum, Sudão } \\
\text { (Cartum, Sudão) } \\
\text { (E2) }\end{array}$ & $\begin{array}{l}151 \text { recém-nascidos } \\
\text { prematuros, peso } \\
(1000-1200 \mathrm{~g}) \text {, idade } \\
\text { gestacional de } 30-37 \\
\text { semanas. }\end{array}$ & $\begin{array}{c}\text { A estimulação } \\
\text { cinestésica tátil } \\
\text { ocorreu em } 3 \text { sessões } \\
\text { de } 15 \text { min, durante } 7 \\
\text { dias consecutivos } \\
\text { (grupo de estudo). }\end{array}$ & $\begin{array}{l}\text { Para análise dos dados (SPSS). O The chi-square } \\
\text { test para analisar as diferenças nas características } \\
\text { mãe- bebê e o teste "t" para analisar as diferenças } \\
\text { entre o grupo de controle e os casos. }\end{array}$ \\
\hline
\end{tabular}


Tabela 2

Características dos estudos avaliados (Continuação)

\begin{tabular}{|c|c|c|c|}
\hline País & População/amostra & $\begin{array}{c}\text { Tempo de } \\
\text { intervenção }\end{array}$ & Análise \\
\hline $\begin{array}{l}\text { Mashhad, Iran } \\
\text { (Mexed, Irã) } \\
\quad(\text { E3) }\end{array}$ & $\begin{array}{l}121 \text { neonatos, com } \\
\text { menos de } 28 \text { dias de } \\
\text { vida, e idade } \\
\text { gestacional inferior a } \\
37 \text { semanas. }\end{array}$ & $\begin{array}{c}\text { A massagem } \\
\text { terapêutica aconteceu } \\
\text { durante uma semana, } \\
4 \text { vezes ao dia, } \\
\text { durante } 5 \text { minutos } \\
\text { (grupo de estudo). }\end{array}$ & $\begin{array}{c}\text { O Chi-square test foi aplicado para determinar os } \\
\text { dados qualitativos. Os dados foram analisados por } \\
\text { meio da one- way, técnica de análise das } \\
\text { variáveis. }\end{array}$ \\
\hline
\end{tabular}

Constata-se que a média da amostra é de 102 prematuros, sendo que todos os neonatos nasceram com idade gestacional acima de 29 semanas, e peso inferior a $1000 \mathrm{~g}$ (E1; E2), contemplando todos os graus de prematuridade. $\mathrm{O}$ tempo de intervenção variou de uma semana a cinco meses de intervenções contínuas. Quanto ao processo de análise, dois artigos usaram a plataforma do SPSS para análise dos dados e todos os artigos se apoiaram em testes para validar ou anular suas hipóteses.

\section{Discussão}

Os resultados dos estudos demonstraram haver associação positiva entre a intervenção promovida e o aumento de peso acelerado nos neonatos prematuros (E3; E2), a alta hospitalar antecipada (E2) e o alívio da dor durante o procedimento clínico (E1). O estudo de Alinejad-Naeini, Mohagheghi, Peyrovi \& Mehran (2014), selecionado para esta revisão, abarca o impacto da posição facilitadora/fetal na dor dos neonatos durante a sucção endotraqueal. A posição facilitadora/fetal é entendida como um método de alinhamento do bebê (Obeidat, Kahalaf, Callister \& Froelicher, 2009), em que o bebê é colocado na posição lateral, com as extremidades flexionadas e aquecidas (Tamez, 2013). Procedimento este, que para Liaw et al. (2012), reduz a dor durante procedimentos externos invasivos e promove ao recémnascido uma sensação de segurança.

A hipótese inicial do estudo de Alinejad-Naeini et al. (2014) foi confirmada, ao ser constatado que o grupo de intervenção, o qual realizou a sucção durante a posição facilitadora/fetal, teve a pontuação da dor menor do que a do grupo que recebeu a sucção sem intervenção. A Escala de Avaliação Comportamental de Brazelton também é um modelo de intervenção terapêutica que avalia diversos aspectos do desenvolvimento neuromotor e neurocomportamental do recémnascido pré-termo e permite a interação paisbebê (Brazelton \& Nuget, 1995; Guedeney \& Lebovici, 1999).

De acordo com Papalia e Feldman (2013), o tato é o primeiro sentido a se desenvolver na criança, sendo que, a sensibilidade cutânea pode ser observada a partir da sexta ou sétima semana de vida, quando o recém-nascido reage aos toques e tende a explorar seu corpo (Oliveira, 2007). Os estudos de Hill, Engle, Jorgensen, Kralik e Whitman (2005) e Menon e McIntosh (2008) sobre as intervenções da posição facilitadora/fetal com neonato prematuro, constataram que esta população sente e compreende a dor de forma mais intensa, se comparado ao neonato a termo.

O manejo da dor, como citado anteriormente por Liaw et al. (2012), promove no neonato prematuro a sensação de segurança e o toque ao recém-nascido, durante os procedimentos da UTI Neonatal, pode auxiliálo na sua adaptação extrauterina (Axelin, Salanterä, \& Lehtonen, 2006). O recémnascido, devido à sua prematuridade, é privado dos cuidados parentais e vivencia um ambiente invasivo na UTIN (Tropiano, 2013). Mesmo afastada destes intensos cuidados parentais, a equipe de profissionais responsáveis pelos cuidados do neonato, pode fazer esse papel de continência (Brum \& Schermann, 2005; Chiodi, Aredes, Scochi \& Fonseca, 2012). 
Os resultados encontrados em Fernandes, Toledo, Campos e Vilelas (2014), reforçam a importância da afetividade no ato de cuidar e salientam o impacto que esse trato emocional tem na vida dos prematuros e dos familiares. Esta forma de cuidado foi descrita nos estudos aqui selecionados (AlinejadNaeini et al. [E1], 2014; Ahmed, et al. [E2], 2015; Saeidi et al. [E3], 2015), os quais corroboram o modelo de apego seguro, descrito por Bowlby (1997), formado através de uma relação afetiva com as principais figuras cuidadoras. Winnicott (1999, citado por Peixoto Junior, 2013), salienta que nem todo nascimento é traumático, se a mãe ou outra figura cuidadora estiver apta a se devotar ao bebê e fizer a sua adaptação ativa, suprindo suas necessidades.

A estimulação cinestésica tátil (ECT) ou massagem terapêutica (MT), contou com a participação das mães (Ahmed et al., 2015). As mães forneceram carícias por todo o corpo do seu bebê prematuro, três vezes por dia, 15 minutos por sessão, durante sete dias, obtendo, assim, resultados significativos no ganho de peso. Os benefícios desta ação vão muito além do físico. Para Winnicott (1999, citado por Peixoto Junior, 2013), as primeiras manifestações de amor são corporais, em que a pessoa do bebê começa a se relacionar com o seu corpo, fazendo assim, a inter-relação de corpo e mente. A presença corporal do outro fornece ao bebê uma integração de diferentes experiências sensoriais, que irão auxiliá-lo a ressignificar de forma imaginária os encontros que ele teve com sua mãe e, gradativamente, irá surgir um corpo psíquico entendido, na teoria winnicotiana, por "corpo-afeto" (Shaffer, 2005).

Outra técnica trazida no estudo de Saeidi, Ghorbani \& Moghadam (2015) é a massagem com óleo de triglicerídeos de cadeia média (MCT), também considerada uma MT (Ahmed et al., 2015). Assim como Winnicott, para Freud (1923/1976, p. 41), “O ego é, primeiro e acima de tudo, um ego corporal". É através da pele que o ego compreenderia o psiquismo, sendo esta pele, primeiramente, um objeto estanho, capaz de reproduzir sensações internas e externas, onde se passaria a reconhecer a "eu/não eu".

Verifica-se, a partir dos resultados dos estudos analisados sistematicamente, que o ganho de peso foi significativo, tanto na pesquisa em que se utilizou da MCT (Saeidi et al. [E3], 2015), quanto na pesquisa da intervenção da massagem sem óleo (Ahmed et al, 2015). Entretanto, o ganho de peso foi maior no grupo de massagem com óleo, do que no grupo de massagem no qual o mesmo não foi administrado. Os achados deste estudo (E3) sugerem que a alimentação transcutânea com massagem terapêutica com óleo, em recém-nascidos prematuros, pode resultar em ganho de peso acelerado nesta faixa etária. As massagens foram realizadas por enfermeiras especializadas (E3), ou por pais (E2). Em ambos estudos, a massagem foi realizada após as refeições.

\section{Considerações finais}

No presente estudo foi possível revisar os artigos científicos sobre a temática da intervenção hospitalar com prematuros. Os achados mostraram similaridade entre a sua população e seus resultados. Observa-se que os estudos abordam intervenções alternativas, que complementam a rotina diária de cuidados e procedimentos ao neonato prematuro. Os procedimentos são simples, de baixo custo, e mobilizam diversos profissionais e, por vezes, os familiares. Ambos visam à promoção da saúde física e, consequentemente, ao utilizarem os cuidados humanizados, auxiliam no desenvolvimento psíquico do bebê, respeitando as suas dimensões biopsicossociais.

Esse estudo ressalta a importância que as intervenções promovidas pelas áreas da saúde têm no impacto gerado ao pré-termo. Analisando sistematicamente os resultados dos estudos sob intervenção hospitalar multiprofissional com prematuros, observouse significativa relação entre o manejo humanizado nos procedimentos de rotina do neonato prematuro hospitalizado, com a sua melhora significativa, no que diz respeito ao 
aumento de peso acelerado nos neonatos prematuros (E3; E2), a alta hospitalar antecipada (E2) e o alívio da dor durante o procedimento clínico (E1).

Os achados dessa pesquisa indicam que, mesmo tratando-se de procedimentos realizados por profissionais da saúde - que não são psicólogos, mas vivenciam a rotina da UTIN - os resultados das massagens com óleo (E3) e sem óleo (E2), bem como a posição facilitadora (E1) corroboram com as práticas da psicologia. Essas intervenções então relacionadas com a construção psíquica saudável do bebê, na promoção de um ambiente seguro, acolhedor e provedor das suas necessidades. Mesmo diante da internação, adversidade esta, que promove o afastamento mãe-bebê, as intervenções buscam aproximar a díade, por meio do contato físico, pele com pele.

Optou-se pela literatura psicanalítica pertinente para discutir os resultados encontrados nos estudos revisados sistematicamente. Enquanto os autores dos estudos pesquisados relacionam as suas intervenções com o ganho de peso nos neonatos prematuros, a alta hospitalar antecipada e o alívio da dor durante o procedimento clínico, a psicologia discute esses achados para além dos benefícios físicos gerados ao prematuro. No campo psicanalítico, a construção do ego e do vínculo perpassam pela dimensão corporal e do vínculo afetivo, utilizando-se do contato físico como um canal para esta integração, pois nos primeiros tempos de vida do bebê, como referiu Freud, o ego é corporal, só posteriormente se tornará psíquico.

Tratam- se de intervenções que envolvem o contato direto com o corpo do neonato, seja por meio das massagens (E2; E3) ou por meio da posição facilitadora (E1) que proporciona conforto ao prematuro. Uma vez que os achados apresentam a construção do ego como corporal, a equipe pode promover estes primeiros cuidados e introduzir os pais neste processo, instruindo-os quanto ao manejo adequado dentro das limitações do bebê prematuro e do contexto hospitalar.

Tendo em vista o exposto, o Método Canguru é apresentado como uma proposta de intervenção, de cunho nacional e internacional, que vem ao encontro dos resultados desse estudo. As estratégias desse método prezam pela humanização dos serviços prestados aos recém-nascidos prematuros e seus familiares. A estratégia promove a construção do apego seguro, incentivo ao aleitamento materno, fortalecimento dos vínculos familiares e desenvolvimento integral desta criança. A Posição Canguru, que integra o método, promove participação ativa dos familiares, em especial da mãe. O mesmo caracteriza-se pelo contato pele a pele, que ocorre de forma gradual. Começa pelo toque (massagem) até a posição canguru (colo) (MS, 2017).

Ainda, é digno de nota, que esta revisão apontou para uma amostra pequena diante da complexidade da temática. Por outro lado, evidencia a escassez de estudos na área, o que limitou análises generalizáveis. Sugerem-se futuras atualizações desta revisão sistemática, seguindo os passos do protocolo aqui utilizado, no intuito de requalificar os resultados encontrados neste estudo $\mathrm{e}$ as intervenções publicadas. Estudos adicionais voltados à mensuração quantitativa das intervenções hospitalares com prematuros são entendidos como necessárias para estimar o reflexo destas intervenções no desenvolvimento do prematuro, no índice de óbitos desta população, e poderiam mensurar o impacto das relações corporais mãe-bebê, nos primórdios da relação para a construção psíquica e na formação do ego do recémnascido.

\section{Referências}

Ahmed, R. G., Suliman, G. I., Elfakey, W. A., Salib, K. M., El-Amin, E. I., Ahmed, W.
A., \& Khalid, K. E. (2015). Effect of tactile kinesthetic stimulation on preterm infants' 
weight and length of hospital stay in Khartoum, Sudan. Saudi Medical Journal, 36(2), 196-199.

doi:10.15537/smj.2015.2.9415.

Alinejad- Naeini, M., Mohagheghi, P., Peyrovi, H., \& Mehran, A. (2014). The Effect of Facilitated Tucking during Endotracheal Suctioning on Procedural Pain in Preterm Neonates: A Randomized Controlled Crossover Study. Global Journal of Health Science, 6(4). doi: 10.5539/gjhs.v6n4p278.

Axelin, A., L. Salanterä S, Lehtonen L. (2006). Facilitated tucking by parents in pain management of preterm infants-a randomized crossover trial. Early Human Development, 82(4), 241-247. doi:10.1016/j.earlhumdev.2005.09.012.

Basiri-Moghadam, M., Basiri-Moghadam, K., Kianmehr, M., \& Jani, S. (2015). The effect of massage on neonatal jaundice in stable preterm newborn infants: a randomized controlled trial. Journal of Pakistan Medical Association, 65(6), 602-60.

Bilotti, C. C., Gomes, E. S., Bianchi, A. B., Bolsoni, L. L. M., Santos, S. M. A., \& Bernuci, M. P. (2016). Método mãe canguru para recém-nascidos de baixo peso: revisão da literatura. Saúde e Pesquisa, 9(3), 587-595. doi:10.17765/1983-1870.2016v9n3p587595.

Bowlby, J. (1990). Formação e rompimento dos laços afetivos (5a. ed.). São Paulo, SP: Martins Fontes.

Bowlby, J. (1997). Formação e rompimento de laços afetivos. São Paulo, SP: Martins Fontes.

Brazelton, T. B., \& Nugent, J. K. (1995). Neonatal Behavioral Assessment Scale $\left(3^{\mathrm{a}}\right.$ ed.). Cambridge: Cambridge, University Press.

Brum, E. H. M. de., \& Schermann, L. (2005). Intervenções frente ao nascimento prematuro: uma revisão teórica. Scientia Medica, 15(1). Recuperado de http://revistaseletronicas.pucrs.br/scientiam edica/ojs/index.php/scientiamedica/article/v iew/1545.
Carvalho, M. R., \& Prochnik, M. (2001). Método mãe-canguru de atenção ao prematuro (Coleção BNDS Social, Vol. 1). Rio de Janeiro, RJ: BNDES.

Charpak N., Figueroa Z., \& Hamel, A. (1999). O método mãe canguru: pais e familiares dos bebês prematuros podem substituir as incubadoras. Rio de Janeiro, RJ: McGrawHill.

Chiodi, L. C., Aredes, N. Del'A., Scochi, C. G. S., \& Fonseca, L. M. M. (2012). Educação em saúde e a família do bebê prematuro: uma revisão integrativa. Acta Paulista de Enfermagem, 25(6), 969-974. doi:10.1590/S0103-21002012000600022.

Cooijmans, K. H. M., Beijers, R. Rovers, A. C., \& Weerth, C. (2017). Effectiveness of skin-to-skin contact versus care-as-usual in mothers and their full-term infants: study protocol for a parallel-group randomized controlled trial. BMC Pediatrics, 17, 154. doi: 10.1186/s12887-017-0906-9.

Costa, K. S. F., Beleza, L. O., Souza, L. M., \& Ribeiro, L. M. (2016). Rede de descanso e ninho: comparação entre efeitos fisiológicos e comportamentais em prematuros. Revista Gaúcha de Enfermagem, 37(esp): e62554. doi:10.1590/1983- 1447.2016.esp.62554.

Duarte, A. D. S., Santos, W. D. S., Silva, L. D. B. D., Oliveira, J. D. D., \& Sampaio, K. J. A. D. J. (2010). Promoção da saúde às genitoras de bebês prematuros: Ação da enfermagem na alta hospitalar. Revista da Rede de Enfermagem do Nordeste, 11(3), 162-170. Recuperado de http://pesquisa.bvs.br/brasil/resource/pt/bde $-18910$.

Fernandes, A., Toledo, D., Campos, L., \& Vilelas, J. M. S. (2014). A Emocionalidade no Ato de Cuidar de Recém-Nascidos Prematuros e Seus Pais: Uma competência do enfermeiro. Pensar Enfermagem, 18 (2), 45-60. Recuperado de http://pensarenfermagem.esel.pt/files/Artig o3_45_60.pdf.

Fundação Osvaldo Cruz: uma instituição a serviço da vida (FIOCRUZ). (2016). Taxa de bebês prematuros no país é quase o dobro do que em países da Europa. 
Recuperado em 18 de Janeiro de 2018 de https://portal.fiocruz.br/pt-

br/content/prematuridade-provocada-porintervencao-medica-chega-40-no-brasil

Freud, S. (1976). O ego e o id. In S. Freud, Edição brasileira das obras psicológicas completas de Sigmund Freud (Vol. 19, pp. 13-83). Rio de Janeiro: Imago. (Artigo original publicado em 1923).

Galvão, T. F., Pansani, T. de S. A., \& Harrad. D. (2015). Principais itens para relatar Revisões Sistemáticas e Meta- análises: A recomendação PRISMA. Epidemiologia e Serviços de Saúde, 24(2). doi: 10.5123/S1679-49742015000200017.

Guedeney, A., \& Lebovici, S. (1999). Intervenções psicoterápicas pais/bebê. Porto Alegre, RS: Artes Médicas Sul.

Hill, S., Engle, S., Jorgensen, J., Kralik, A., \& Whitman, K. (2005). Effects of facilitated during routine care of infants born preterm. Pediatric Physical Therapy, 17(2), 158163. doi: 10.1097/01.PEP.0000163097.38957.EC

Liaw, J. J, Yang, L., Katherine Wang, K. W., Chen, C. M., Chang, Y. C., \& Yin, T. (2012). Non- nutritive sucking and facilitated tucking relieve preterm infant pain during heel- sick procedures: A prospective, randomized controlled crossover trial. International Journal of Nursing Studies, 49(3), 300-309. doi: 10.1016/J.iJnurstu.2011.09.017.

Ministério da Saúde. Gabinete do Ministro. (2011). PORTARIA N ${ }^{\circ} 1.459$, DE 24 DE JUNHO DE 2011: Instituto no âmbito do Sistema Único de Saúde - SUS - a Rede Cegonha. Recuperado de http://bvsms.saude.gov.br/bvs/saudelegis/g m/2011/prt1459_24_06_2011.html

Ministério da Saúde. Secretaria de Atenção à Saúde. Departamento de Ações Programáticas Estratégicas. (2014). Atenção à Saúde do Recém-Nascido: Guia para os Profissionais de Saúdel Ministério da Saúde. Secretaria de Atenção à Saúde. Departamento de Ações Programáticas Estratégicas. Brasília: Editora do Ministério da Saúde. 2.ed.
Ministério da Saúde. Secretária de atenção à Saúde. Departamento de Ações Programáticas Estratégicas. (2017). Atenção à Saúde do Recém-Nascido: Guia para os Profissionais de Saúdel Ministério da Saúde. Secretaria de Atenção à Saúde. Departamento de Ações Programáticas Estratégicas. Brasília: Editora do Ministério da Saúde. 3. ed.

Menon, G., \& McIntosh, N. (2008). How should we manage pain in ventilated neonates? Neonatology, 93(4), 316 -23. doi: 10.1159/000121458.

Moore, E.R., Bergman, N., Anderson, G.C., \& Medley, N. (2016). Early skin-to-skin contact for mothers and their healthy newborn infants. Cochrane Pregnancy and Childbirth Group, 11, 1-121. doi: 10.1002/14651858.CD003519.pub4.

O’Toole, A., Francis, K., \& Pugsley, L. (2017, April 8). Does Music Positively Impact Preterm Infant Outcomes?. Advances in Neonatal Care, 17(3), 192-202. doi: 10.1097/ANC.0000000000000394

Obeidat, H., Kahalaf, I., Callister, L. C., \& Froelicher, E. S. (2009). Use of facilitated tucking for no pharmacological pain management in preterm infants: A systematic review. Journal of Perinatal Neonatal Nursing, 23(4), 372-377. doi: 10.1097/JPN.0b013e3181bdcf77

Oliveira, M. E. (2007). A poesia de cuidar do recém-nascido pré-termo: uma conexão entre o sensível, o intuitivo e o científico (Tese de Doutorado). Universidade Federal de Santa Catarina, Florianópolis, Santa Catarina. Recuperado de https://repositorio.ufsc.br/xmlui/handle/123 $\underline{456789 / 89906}$

Papalia, D. E. \& Feldman, R. D. (2013). Desenvolvimento Humano. Porto Alegre, RS: AMGH.

Peixoto Junior, C. A. (2013). Sobre o corpo afeto em espinosa e Winnicott. Revista Epos, 4(2). Recuperado de http://pepsic.bvsalud.org/scielo.php?script= sci_arttext\&pid=S2178700X2013000200003

Penalva, O., \&amp; Salomão, J. (2006). Estudo descritivo do perfil clínico- 
nutricional e doseguimento ambulatorial de recém-nascidos prematuros atendidos no Programa Método Mãe-Canguru. Jornal de Pediatria, 82(1), 33-39.

Penalva, O., \& Schwartzman, J. S. (2006).

Estudo descritivo do perfil clíniconutricional e do seguimento ambulatorial de recém-nascidos prematuros atendidos no Programa Método Mãe-Canguru. Jornal de Pediatria, 82(1), 33-39. doi:

10.2223/JPED.1434.

Portal da Saúde. (2017). Ministério da Saúde Lança Estratégia para Reduzir Mortalidade Neonatal Recuperado de http://portalsaude.saude.gov.br/index.php/o -ministerio/principal/secretarias/sas/sasnoticias/29023-ministerio-da-saude-lancaestrategia-para-reduzir-mortalidadeneonatal.

Rolim, K. M. C., Vidal, A. F., Mariano, M. A., Campos, A. C. S, \& Frota, M. A. (2008). Percepção das mães sobre aleitamento em prematuros da unidade canguru de uma maternidade de Fortaleza - CE. Revista RENE Fortaleza, 9(2), 54-63. Recuperado de

http://www.periodicos.ufc.br/rene/article/vi ew/4998.

Saeidi, R., Ghorbani, Z., \& Moghadam, A. S. (2015). The Effect of Massage with Medium- Chain Triglyceride Oil on Weight Gain in Premature Neonates. Acta Medica Iranica 2015. 53(2), 134-138. Recuperado de

http://acta.tums.ac.ir/index.php/acta/article/ view/4922.

Shaffer, D. R. (2005). Desenvolvimento e personalidade da criança. São Paulo, SP:

Pioneira. Thomson Learning.

Sharma, A. (2016). Efficacy of early skin-toskin contact on the rate of exclusive breastfeeding in term neonates: a randomized controlled trial. African Health Sciences, 16(3), 790-797. doi: 10.4314/ahs.v16i3.20.

Sharma, D., Murki, S., \& Oleti, T. P. (2016). To compare cost effectiveness of 'Kangaroo Ward Care' with 'Intermediate intensive care' in stable very low birth weight infants (birth weight $<1100$ grams): a randomized control trial. Italian Journal of Pediatrics, 42(64). doi:10.1186/s13052016-0274-3.

Souto, D. S., Jager, M. E., Pereira, A. S., \& Dias, A. C. G. (2014). Método canguru e aleitamento materno: uma revisão integrativa da literatura nacional. Revista Ciência e Saúde, 7(1), 35-46. doi: 10.15448/1983-652X.2014.1.14519

Spehar, M. C., \& Seidl, E. M. F. (2013). Percepções maternas no Método Canguru: contato pele a pele, amamentação e autoeficácia. Psicologia em Estudo, 18(4), 647-656. doi: 10.1590/S141373722013000400007.

Tamez, R. (2013). Enfermagem na UTI Neonatal (5a ed). Rio de Janeiro, RJ: Guanabara Koogan.

Tropiano, L. M. C. C. (2013). Afetividade nas interações mãe-bebê prematuro no primeiro contato físico (Dissertação de mestrado). Universidade Presbiteriana Mackenzie, São Paulo, São Paulo.

Recuperado de http://tede.mackenzie.br/jspui/handle/tede/1 624\#preview-link0

Yates, C.C., Mitchell, A. J., Booth, M. Y., Williams, D. K., Lowe, L. M. \& Whit Hall, R.(2014). The effects of massage therapy to induce sleep in infants born preterm. Pediatric Physical Therapy, 26(4), 405- 410. doi: 10.1097/PEP.0000000000000081.

Winnicott, D. W. (1988). O ambiente e os processos de maturação. Porto Alegre, RS: Artes Médicas.

World Health Organization [WHO]. (2015). Recommendations on interventions to improve preterm birth outcomes.

Recuperado em 29 de outubro de 2017 de http://www.who.int/mediacentre/factsheets/ fs363/en/

World Health Organization [WHO]. (2017). Preterm Birth. Recuperado em 18 de janeiro de 2018 de http://www.who.int/mediacentre/factsheets/ fs363/en/ 


\section{Dados sobre os autores:}

- Carine Tabaczinski: Graduada no Curso de Psicologia, Faculdade Meridional- IMED.

- Denice Bortolin: Graduada em Psicologia pela Universidade de Passo Fundo/UPF (2004); Especialização em Psicologia Clínica: Psicoterapia de Orientação Psicanalítica pela UPF (2008); Mestrado em Educação pela UPF (2011); Doutoranda em Psicologia Clínica na Universidade do Vale do Rio dos Sinos/UNISINOS (2014). Professora Adjunta da Faculdade Meridional-IMED no Curso de Psicologia.

- Luiz Ronaldo Freitas de Oliveira: Psicólogo, Professor e Coordenador de Graduação na IMED. Doutor em Psicologia pela Universidade do Vale do Rio dos Sinos-UNISINOS (2016); Mestre em Psicologia pela UNISINOS (2010); Especialização em Psicologia Clínica pela IMED (2007); Graduado em Filosofia, Teologia e Psicologia. Além da atividade docente atende em consultório particular. 